\title{
An Assessment of Artificial Compressibility and Pressure Projection Methods for Incompressible Flow Simulations
}

D. Kwak and C. Kiris

NASA Ames Research Center, Moffett Field, CA 94035, USA

e-mail: dkwak@mail.arc.nasa.gov, kiris@nas.nasa.gov

Key Words: Incompressible Flow, Artificial Compressibility Method, Pressure Projection Method

\begin{abstract}
Performance of the two commonly used numerical procedures, one based on artificial compressibility method and the other pressure projection method, are compared. These formulations are selected primarily because they are designed for three-dimensional applications. The computational procedures are compared by obtaining steady state solutions of a wake vortex and unsteady solutions of a curved duct flow. For steady computations, artificial compressibility was very efficient in terms of computing time and robustness. For an unsteady flow which requires small physical time step, pressure projection method was found to be computationally more efficient than an artificial compressibility method. This comparison is intended to give some basis for selecting a method or a flow solution code for large threedimensional applications where computing resources become a critical issue.
\end{abstract}

\section{Introduction}

Various procedures can be selected for simulating incompressible flows depending on the choice of formulations, variables, discretization and iterative schemes. In the present study, performance of the two procedures commonly used for threedimensional applications are compared: the first one based on an artificial compressibility method and the second one on a pressure projection method.

The artificial compressibility method takes advantage of the advances made in conjunction with compressible flow computations. This approach relaxes the requirement of enforcing mass conservation equation rigorously at each time iteration, however, at the expense of introducing an artificial wave phenomenon. This approach can be viewed as a special case of a preconditioned compressible flow formulation. However, the computational efficiency is in general better than that of compressible flow solvers at the incompressible limit. This approach has been shown to be very robust in a wide range of applications [Kwak et. al (1986), Rogers et. al (1991)].

The first primitive variable method for incompressible flow was developed by Harlow and Welch (1965) using pressure projection. Numerous variants have been developed since. In this method, the pressure is used as a mapping parameter to satisfy the continuity equation. The usual computational procedure involves choosing the pressure field at the current time step such that continuity is satisfied at the next time step. The time step is advanced in multiple steps (fractional step) which is computationally convenient. However, governing equations are not coupled as in an artificial compressibility approach. This will affect the robustness and limit the maximum allowable time step size. Since this approach is time accurate, there are cases where the fractional step solver is computationally more efficient compared to the artificial compressibility method [Rosenfeld et. al (1991), Kiris and Kwak (1996)].

Various numerical algorithms associated with these methods have been developed along with accompanying flow solvers. In three-dimensional applications computing 
time requirement is one of the key issues in selecting flow solvers. In the present study it is intended to give some bench mark comparisons on computing efficiencies between the two methods discussed above.

\section{Artificial Compressibility vs. Pressure Projection Formulations}

Governing equations for these two methods are presented here to facilitate the discussion of results. The artificial compressibility method by Chorin (1967) can be written as

$$
\begin{aligned}
& \frac{1}{\beta} \frac{\partial p}{\partial t}+\frac{\partial u_{i}}{\partial x_{i}}=0 \\
& \frac{\partial u_{i}}{\partial t}=-\nabla p+h_{i}
\end{aligned}
$$

where usual conventions are used with $h_{i}$ representing convection and viscous terms. At steady state the pressure term in equation (1) drops out and thus incompressibility is recovered. For time accurate computations, this has to be repeated at each time level to maintain incompressibility at each time step.

The pressure projection procedure on the other hand requires solving the following pressure Poisson equation to satisfy the continuity at the next time level:

$$
\nabla^{2} p=\frac{\partial h_{i}}{\partial x_{i}}-\frac{\partial}{\partial t} \frac{\partial u_{i}}{\partial x_{i}}
$$

Numerically intermediate velocity field is computed first then pressure correction is computed using the pressure Poisson equation. Once the pressure correction is computed, new pressure and velocities are calculated. In this approach the Poisson solver portion is usually the most expensive part of the computational procedure. Therefore, accelerating convergence for Poisson solver has been the focal point of many previous studies.

In the present paper, the two methods are compared using solvers developed at NASA Ames Research Center. The artificial compressibility method is represented by INS3D-UP code which utilizes line relaxation in conjunction with upwind differencing. The pressure projection method is represented by INS3D-FS which utilizes RungeKutta time stepping in conjunction with finite volume scheme on staggered grid arrangement. Code performance is a function of many factors such as iterative scheme, spatial differencing, coding and choice of parameters like artificial compressibility. However, the performance figures presented next will provide some indications on what one might expect from similar codes in computing steady-state or time-accurate problems encountered in engineering practices.

\section{Computed Results}

Two test problems are selected: a wake vortex problem for steady-state solutions, and a curved duct flow with pulsatile inflow condition. The wake vortex problem is selected because it represents aerodynamic and hydrodynamic problems involving wingtip. The high gradient in the flow field poses significant challenges for computing this flow. The pulsatile flow in curved duct is chosen to represent internal flows frequently encountered in propulsion and bioengineering. 
In Figure 1, tip vortex generated from a wing is shown from an earlier numerical and experimental study by Dacles-Mariani et. al (1993). A round tip NACA 0012 wing is placed at 10 degrees angle of attack at $R_{e}=4.6 \times 10^{6}$. The present computational domain starts at the trailing edge of this wing. The two codes are compared for obtaining steady-state solution using $35 \times 81 \times 81$ grid. The flow field has a highly concentrated vortical core region which poses challenges in implementing the right turbulence model and grid distribution. In Figure 2, convergence history using INS3DUP and INS3D-FS is shown. For steady-state solutions, INS3D-UP code required 2 hours of Cray C-90 CPU time while INS3D-FS needed 4.2 hours to lower the residual down to $10^{-13}$. The pressure projection required smaller time step to maintain the projection accuracy which resulted in approximately twice more iterations than the artificial compressibility method. In Figure 3, results using various production terms in the Baldwin-Barth turbulence model (denoted as BB) are compared using INS3D-FS. Also shown is a coarse-grid solution using lower order spatial differencing. The corresponding velocity profiles at $x / c=1.447$ are shown in Figure 4 . Figure 4 shows that the large differences among different experiments in Figure 3 are mainly in the core region, and in the outer region, the computed velocity compares well with experiment. This study shows the relative importance of selecting the right turbulence model as well as grid resolution in computing tip vortex flow. In Figure 5, the velocity magnitudes from the two codes and experiment are plotted at $x / c=1.587$. Full details of this study can be found in Dacles-Mariani et. al (1993) and Kiris and Kwak (1996).

For an unsteady test, a square duct with 120 degree bend was chosen. The inflow as shown in Figure 6 represents a typical unsteady flow from an animal heart. The Reynolds number based on average inflow velocity is 625 .

To get time-accurate solution for this problem using INS3D-UP, the solver has to be subiterated at each time level until a reasonable divergence free velocity is attained. In the present case, the number of subiterations was limited to 15 . Realistically, if more subiteration is required, decreasing time step size while maintaining low number of subiteration is generally more economical. As shown in Table 1, with 200 time steps in each pulsating cycle, the solution still carries relatively large mass conservation error of up to 5 percent. Since the artificial compressibility does not require divergence free condition, the computational procedure produced converging solution. When the total number of time step per cycle is increased to 500, the resulting unsteady solution maintained mass conservation within a reasonable accuracy. The total time required for this case was 10.4 hours of Cray C-90 CPU time.

The same case was run using INS3D-FS next. When the time step size is large with the total number of time step of 200 , the projection error was too large. The velocity estimated at intermediate step and the subsequent pressure projection to the new velocity produced mass conservation error which failed to produce time accurate result. By decreasing the time step size which increases the total number of time step to 500 , the solution maintained mass conservation and the desired time accuracy desired. Since the projection method does not require expensive subiterations as in the case of an artificial compressibility method, the computational efficiency was better than the result of INS3D-UP.

The CPU time required per point per iteration varies depending on the choice of number of sweeps in line relaxation or iterative schemes. The comparison of 
performance is better represented by the total time required for obtaining converged solutions.

Table 1. Performance comparison for unsteady flow in curved duct flow using $51 \times 41 \times 41$ grid.

\begin{tabular}{|l|l|l|l|l|l|l|}
\hline Code & $\begin{array}{l}\text { Memory } \\
\text { Words } \\
\text { /point }\end{array}$ & $\begin{array}{l}\text { CPU/itera } \\
\text { tion/point, } \\
\mu \mathrm{sec}\end{array}$ & $\begin{array}{l}\text { No.of } \\
\text { Sub- } \\
\text { iteration }\end{array}$ & $\begin{array}{l}\text { Total No. } \\
\text { of Time } \\
\text { Step }\end{array}$ & $\begin{array}{l}\text { Total } \\
\text { CPU } \\
\text { (C-90 hr) }\end{array}$ & Remarks \\
\hline INS3D-UP & 40 & 60 & 15 & 200 & 4.2 & $\begin{array}{l}5 \% \text { error in mass } \\
\text { conservation }\end{array}$ \\
\hline INS3D-UP & 40 & 60 & 15 & 500 & 10.4 & \\
\hline INS3D-FS & 60 & 120 & $\mathrm{n} / \mathrm{a}$ & 200 & $\mathrm{n} / \mathrm{a}$ & Projection error too big \\
\hline INS3D-FS & 60 & 120 & $\mathrm{n} / \mathrm{a}$ & 500 & 1.4 & Implicit 3-step R-K \\
\hline
\end{tabular}

In Figure 6, the results from these two codes are compared by showing the velocity vectors in mid-plane of the duct. This figure does not provide a good quantitative comparison. However, considering that the mass flow rate is conserved, the flow field inside the duct compares well between the two codes.

\section{Concluding Remarks}

The computational results show performance of the two incompressible flow methods. For steady state solutions, an artificial compressibility method combined with compressible flow algorithms is shown to be very efficient. For time-accurate solutions, on the other hand, the subiteration procedure required of an artificial compressibility method tends to be expensive. The pressure projection method offers a better alternative for unsteady flow computations. The examples presented here are only for Reynolds averaged Navier-Stokes type solutions, and did not address features required for a real fine mesh calculations like Large Eddy Simulations.

\section{References}

Chorin, A. J., "A Numerical Method for Solving Incompressible Viscous Flow Problems," J. Comp. Phys., Vol. 2, pp.12-26, 1967.

Dacles-Mariani, J., Rogers, S., Kwak, D., Zilliac, G. and Chow, J. 'A Computational Study of Wingtip Vortex Flowfield,' AIAA Paper 93-3010, AIAA 24th Fluid Dynamics Conference, Orlando, FL, July 6-9, 1993.

Harlow, F. H. and Welch, J. E., "Numerical Calculation of Time-Dependent Viscous Incompressible Flow with Free Surface," Phys. Fluids, Vol. 8, No. 12, pp. 2182-2189,1965.

Kiris, C. and Kwak, D., 'Numerical Solution of Incompressible Navier-Stokes Equations Using a Fractional-Step Approach,' AIAA Paper 96-2089, AIAA 27th Fluid Dynamics Conference, New Orleans, LA, June 17-20, 1996

Kwak, D., Chang, J. L. C., Shanks, S. P., and Chakravarthy, S., 'An Incompressible NavierStokes Flow Solver in Three-Dimensional Curvilinear Coordinate Systems Using Primitive Variables,' AIAA J., vol 24, No. 3, 390-396, Mar. 1986.

Rogers, S. E., Kwak, D. and Kiris, C., 'Steady and Unsteady Solutions of the Incompressible Navier-Stokes Equations,' AIAA J. vol. 29, No. 4, pp 603-610, April, 1991.

Rosenfeld, M., Kwak, D., and Vinokur, M., 'A Solution Method for Únsteady, Incompressible Navier-Stokes Equations in Generalized Curvilinear Coordinate Systems,' J. Comp. Phys. vol 94, No. 1, May 1991, pp102-137. 

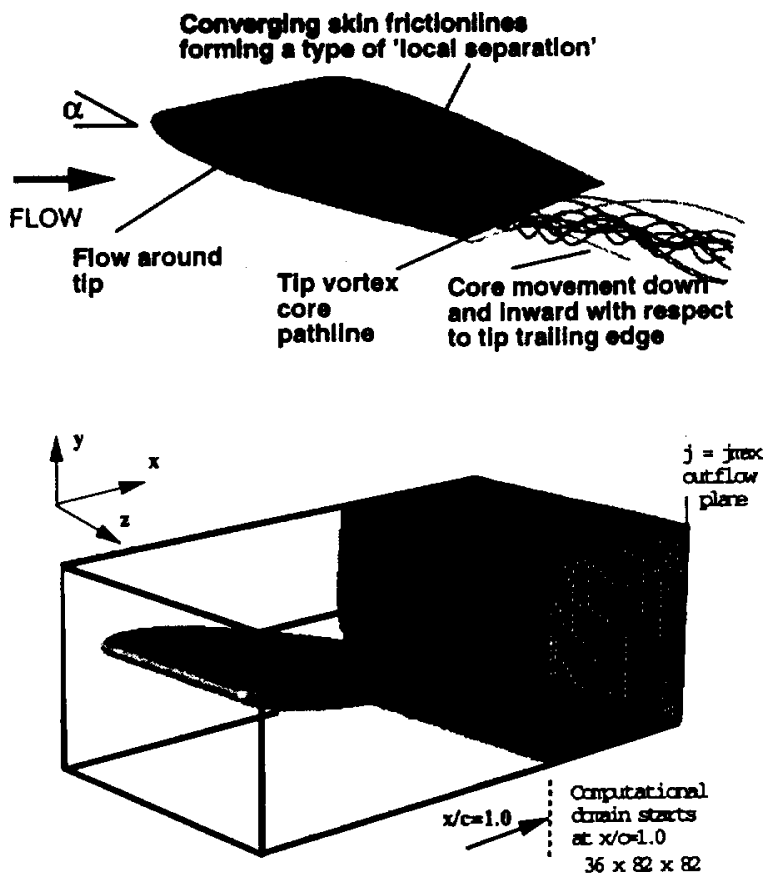

Figure 1 : Features of a wing tip vortex flowfield, and computational domain for wake vortex flow calculations.
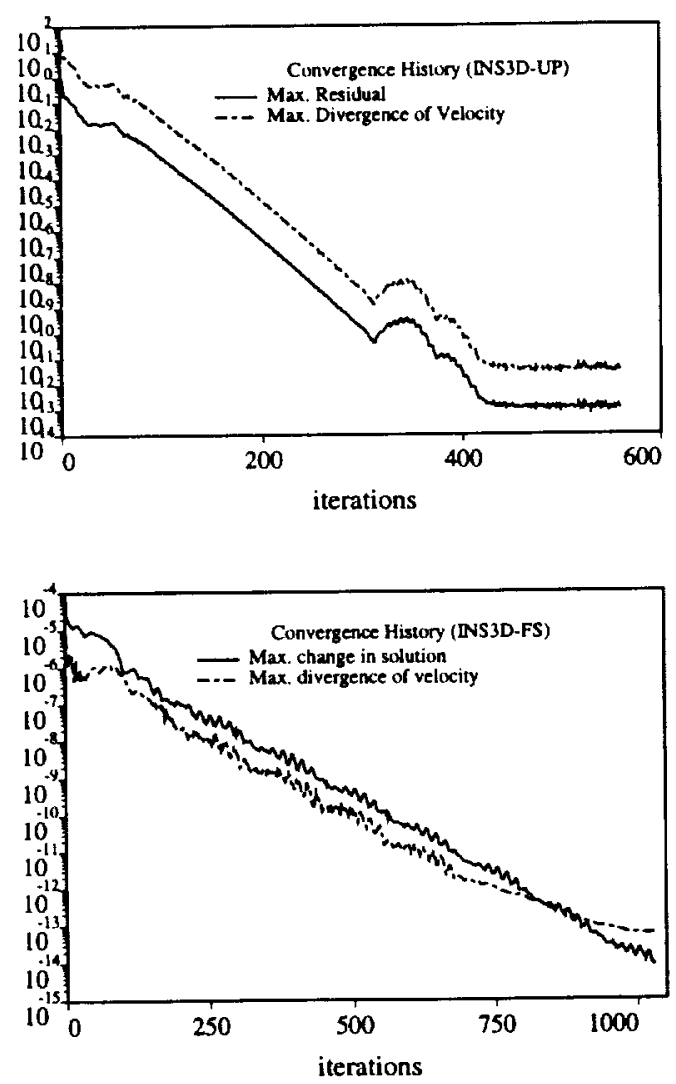

Figure 2 : Convergenge history for wake vortex case.

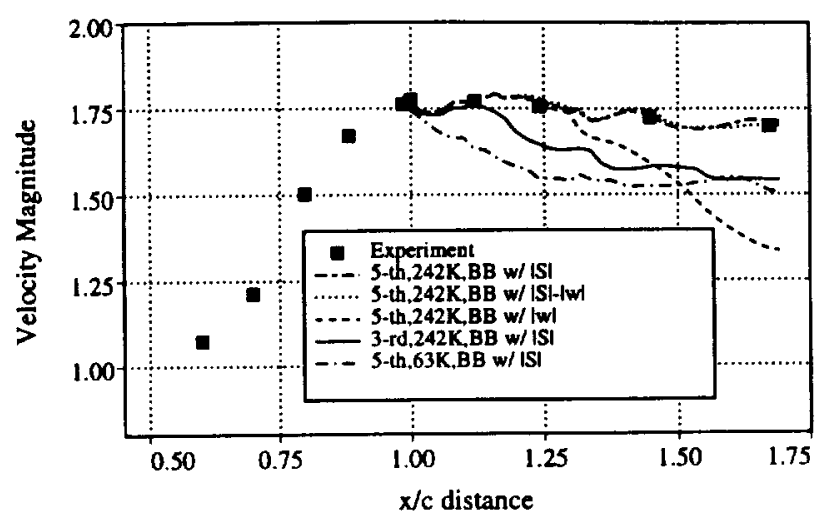

Figure 3 : Axial progression of velocity magnitude along vortex coreline (INS3DFS).

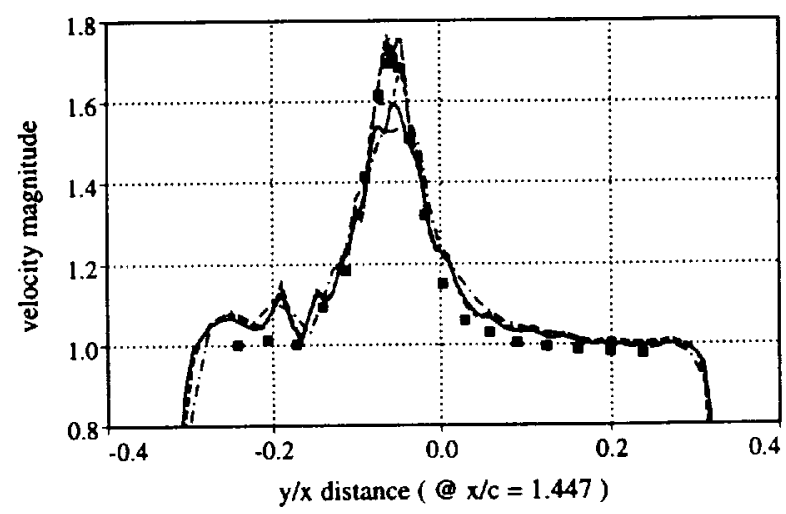

Figure 4 : Comparison of velocity magnitude across wake vortex (INS3D-FS).

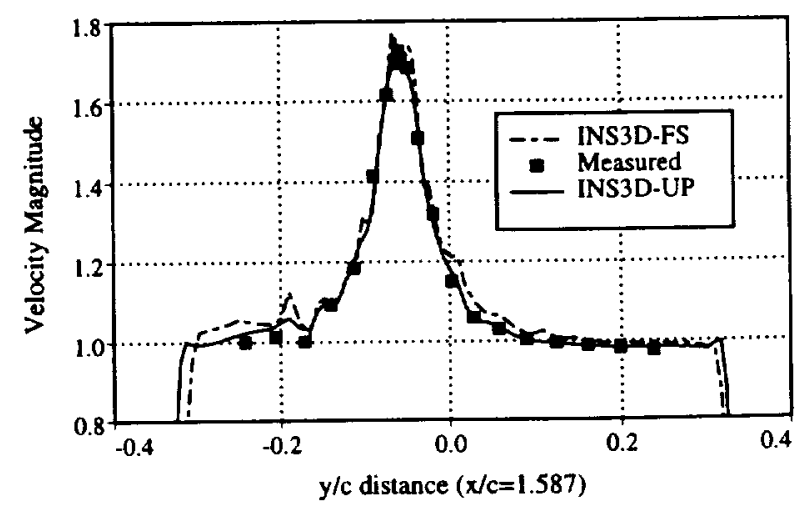

Figure 5 : Comparison of velocity magnitudes from computed results of INS3DFS and of INS3D-UP. 

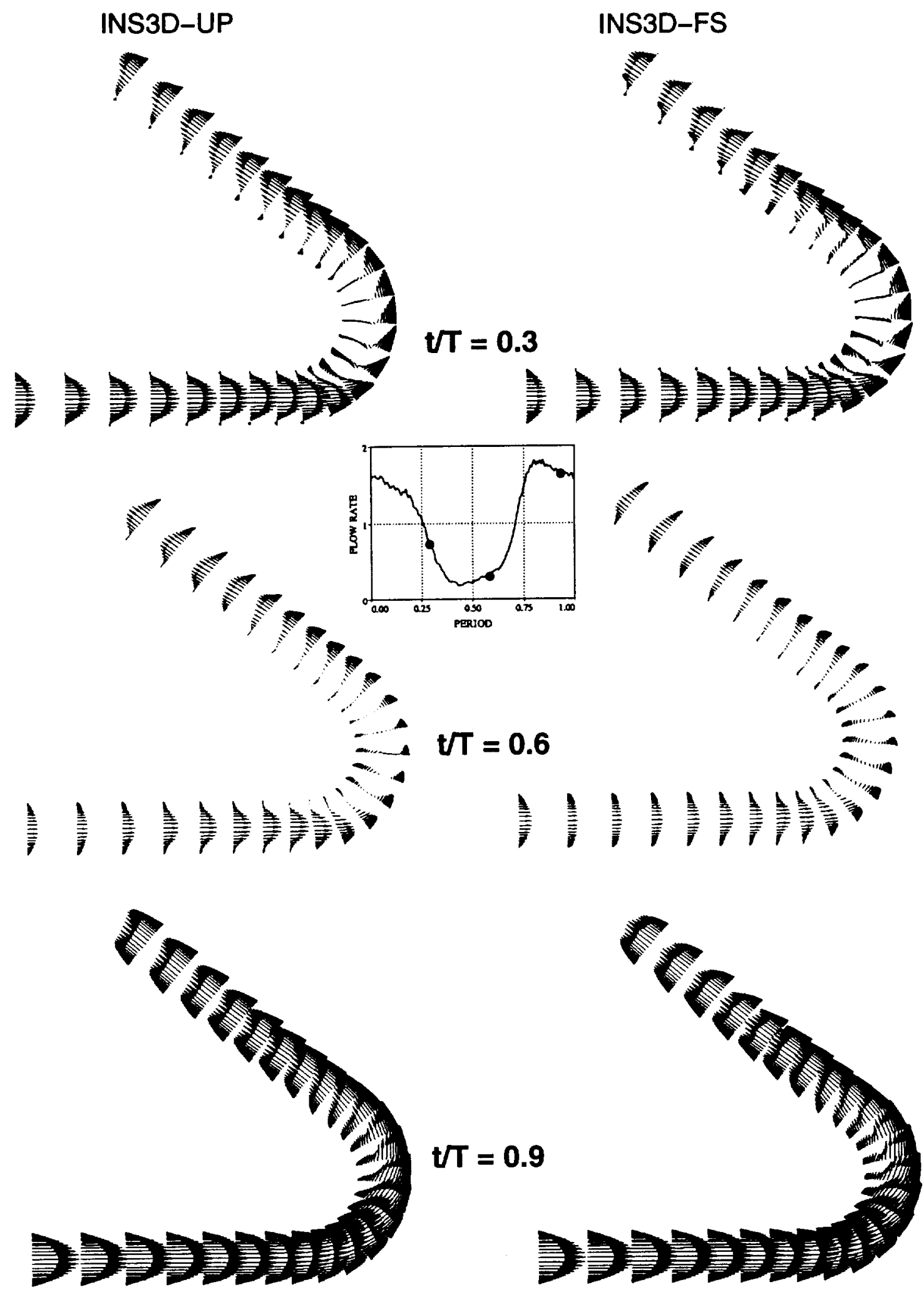

Figure 6. Velocity vectors at centerplane of 120-degree suare duct. Unsteady inflow profile is imposed from animal measurements. 